\title{
An axiomatic definition of cardinality for finite interval-valued hesitant fuzzy sets
}

\author{
Pelayo Quirós ${ }^{1}$ Pedro Alonso ${ }^{1}$ Irene Díaz $^{2}$ Vladimír Janišs ${ }^{3}$ \\ ${ }^{1}$ Department of Mathematics, University of Oviedo, Spain. e-mails: \{uo205956,palonso\}@uniovi.es \\ ${ }^{2}$ Department of Computer Science, University of Oviedo, Spain. e-mail: sirene@uniovi.es \\ ${ }^{3}$ Department of Mathematics, Matej Bel University, Slovak Republic. e-mail: vladimir.janis@umb.sk
}

\begin{abstract}
Recently, some extensions of the classical fuzzy sets are studied in depth due to the good properties that they present. Among them, in this paper finite interval-valued hesitant fuzzy sets are the central piece of the study, as they are a generalization of more usual sets, so the results obtained can be immediately adapted to them.

In this work, the cardinality of finite intervalvalued hesitant fuzzy sets is studied from an axiomatic point of view, along with several properties that this definition satisfies, being able to relate it to the classical definitions of cardinality given by Wygralak or Ralescu for fuzzy sets.
\end{abstract}

Keywords: fuzzy sets, hesitant fuzzy sets, intervalvalued hesitant fuzzy sets, cardinality.

\section{Introduction}

Grattan-Guinnes in [7] introduced the notion of hesitancy using the name of set-valued fuzzy set. Later, Torra introduced the hesitant fuzzy logic in 2009 (see $[15,16]$ ) as an intermediate step between type-2 fuzzy sets and interval-valued fuzzy sets. This type of sets assigns a subset of the interval $[0,1]$ to each element instead of a fuzzy set, which makes them more manageable than type-2 fuzzy sets. Decision making is the main application of the hesitant fuzzy logic, where the opinion of different experts are summarized in a single set (a hesitant fuzzy set).

Several extensions of the hesitant fuzzy sets have been defined lately (see [13]). Among them, interval-valued hesitant fuzzy sets introduced by Chen et al. in 2013 (see [3]), whose membership function assigns a union of disjoint closed subintervals of $[0,1]$, or finite interval-valued fuzzy sets $[9]$ that restricts the definition introduced by Chen to finite union.

For crisp sets, the concept of cardinality is intuitive and easy to define mathematically. However, for fuzzy sets different definitions have been proposed by several authors. De Luca and Termini in 1972 (see [6]) defined the basic $\sigma$-count cardinality. Ralescu proposed in 1995 (see [12]) the concepts of fuzzy and non-fuzzy cardinality. From an axiomatic point of view, Wygralak gives a definition of scalar cardinality for fuzzy sets in 2003 (see [17]).
The aim of this paper is to study the concept of cardinality for finite interval-valued hesitant fuzzy sets from an axiomatic point of view. After providing an axiomatic definition, some properties of these cardinalities are shown. In particular, two cardinalities are studied as they present good properties when restricted to fuzzy sets. The first one matches with $\sigma$-count cardinality given by De Luca and Termini [6], and the second one with the nonfuzzy cardinality defined by Ralescun [12]. Other results around the axiomatic definition of cardinality for finite interval-valued hesitant fuzzy sets are studied, where some properties of this cardinality are shown.

The remainder of the paper is set out as follows: Section 2 presents preliminary concepts. Section 3 is focused on the new definition of cardinality for finite interval-valued hesitant fuzzy sets and related results. In Section 4 the main conclusions of this work are highlighted.

\section{Preliminaries}

In this section, the necessary concepts to follow the paper are given. The definitions that are about to be shown, are well known and can be found in several sources, such as [8]. All these definitions, are important to compare both logics, fuzzy and hesitant, and to understand the motivation of the second one.

The definition of fuzzy set must be the first one to be presented, as it is the basis of all the fuzzy logic and the consequent studies. It was given for the first time by Zadeh (see [20]). Given a fuzzy set $A$, its membership function is denoted by $\mu_{A}$. Furthermore, the set of all fuzzy sets in $X$ is denoted by $F S(X)$.

In the following parts of the paper, the concepts of intersection and union of fuzzy sets will be necessary. There are several possibilities to choose. In this work, the standard ones are selected, which are given as follows:

Definition 2.1 Given $X$ a non-empty set and $A, B \in F S(X)$, then:

$$
\begin{aligned}
& \mu_{A \cup B}(x)=\max \left\{\mu_{A}(x), \mu_{B}(x)\right\}, \forall x \in X, \\
& \mu_{A \cap B}(x)=\min \left\{\mu_{A}(x), \mu_{B}(x)\right\}, \forall x \in X .
\end{aligned}
$$


The interval-valued fuzzy sets are a generalization of the fuzzy sets, where each point is associated to an interval instead of just one value. This concept was originally developed by Sambuc (see [14]).

Definition 2.2 Let $X$ be a non-empty set. Given the membership function:

$$
\mu_{A}: X \rightarrow L([0,1])
$$

where $L([0,1])$ denotes the family of all closed subintervals of $[0,1]$, then, the set $A=\left\{\left(x, \mu_{A}(x)=\right.\right.$ $\left.\left.\left[\mu_{A}(x)^{L}, \mu_{A}(x)^{U}\right]\right) \mid x \in X\right\}$ is an interval-valued fuzzy set in $X$.

The set of all interval-valued fuzzy sets in $X$ is denoted by $I V F S(X)$.

Hesitant fuzzy logic was recently defined by Torra in $[15,16]$, although the first introduction of this concept was made by Grattan-Guinnes in [7], under the name of set-valued fuzzy set. Nevertheless, unlike Grattan-Guinness, Torra provided functional definitions of union and intersection for them. Several papers related to this logic have been published, such as [2], where the basic concepts can be found.

Let $\mathcal{P}([0,1])$ denote the family of subsets of the closed interval $[0,1]$. A typical hesitant fuzzy set is defined as follows (see $[1,2])$ :

Definition 2.3 Let $X$ be a non-empty set and $\mathbb{H} \subset$ $\mathcal{P}([0,1])$ the set of all finite non-empty subsets of the interval $[0,1]$. Given the membership function:

$$
\mu_{A}: X \rightarrow \mathbb{H}
$$

then, the set $A=\left\{\left(x, \mu_{A}(x)\right) \mid x \in X\right\}$ is a typical hesitant fuzzy set in $X$.

The set of all typical hesitant fuzzy sets in $X$ is denoted by $T H F S(X)$.

The membership function of a typical hesitant fuzzy set gives for each element of $X$ a finite subset of the interval $[0,1]$. However, a generalization has been recently developed by Pérez et al. (see [9]), the finite interval-valued hesitant fuzzy sets. This type of sets replaces finite subsets by subsets which are generated by a union of a finite number of closed intervals.

Before providing the definition of this new kind of sets, we introduce the notion of a finitely generated set, and some related concepts that are necessary in the forthcoming results.

Definition 2.4 Let $n \in \mathbb{N}$. The class of $n$-finitely generated sets in $[0,1]$ is given by

$$
\begin{aligned}
F G_{n}([0,1])=\{ & \left\{\subseteq[0,1] \mid I=\bigcup_{i=1}^{n} I_{i}\right. \\
& \text { with } \left.I_{i} \cap I_{j}=\emptyset, \forall i \neq j\right\},
\end{aligned}
$$

where $I_{i} \in L([0,1]), \forall i=1, \ldots, n$.
The class of finitely generated sets in $[0,1]$ is given by

$$
F G([0,1])=\bigcup_{n=1}^{\infty} F G_{n}([0,1])
$$

Some operations between finitely generated sets were given by Chen et al. in [4]. Among them, the most important ones for the study of the cardinality are the union and intersection.

Definition 2.5 Let $I, J \in F G([0,1])$ such that

$I=\bigcup_{i=1}^{n_{I}} I_{i}=\bigcup_{i=1}^{n_{I}}\left[I_{i}^{L}, I_{i}^{U}\right]$ and $J=\bigcup_{i=1}^{n_{J}} J_{i}=\bigcup_{i=1}^{n_{J}}\left[J_{i}^{L}, J_{i}^{U}\right]$,

where $I_{i}$ and $J_{i}$ are pairwise disjoint closed subintervals of $[0,1]$ respectively. Then:

$$
\begin{aligned}
I \vee J= & \left\{\left[\max \left(I_{i}^{L}, J_{j}^{L}\right), \max \left(I_{i}^{U}, J_{j}^{U}\right)\right] \mid\right. \\
& \left.\forall i=1, \ldots, n_{I}, \forall j=1, \ldots, n_{J}\right\}, \\
I \wedge J= & \left\{\left[\min \left(I_{i}^{L}, J_{j}^{L}\right), \min \left(I_{i}^{U}, J_{j}^{U}\right)\right] \mid\right. \\
& \left.\forall i=1, \ldots, n_{I}, \forall j=1, \ldots, n_{J}\right\} .
\end{aligned}
$$

We have developed the next proposition related to intersection of finitely generated sets, as in the forthcoming section, it is an important result.

Proposition 2.6 Let $I, J$ two $F G([0,1])$ such that $I \wedge J=\emptyset$, then $I=\emptyset$ or $J=\emptyset$.

After these prior concepts, the definition of a finite interval-valued hesitant fuzzy set is given as follows (see [4]).

Definition 2.7 Let $X$ be a non-empty set. Given the membership function:

$$
\mu_{A}: X \rightarrow F G([0,1]),
$$

then, the set $A=\left\{\left(x, \mu_{A}(x)\right) \mid x \in X\right\}$ is a finite interval-valued hesitant fuzzy set in $X$.

The set of all finite interval-valued hesitant fuzzy sets in $X$ is denoted by $\operatorname{IVHFS}(X)$.

For every finite interval-valued hesitant fuzzy set, and for each point $x \in X$, the value of the membership function consists of $n_{x}$ closed intervals that generates the finitely generated set. Obviously, in a finite interval-valued hesitant fuzzy set, some of the closed subintervals can be degenerated, i.e., singletons. If all the intervals are degenerated, then we recover typical hesitant fuzzy sets.

Intersection and union definitions for finite interval-valued hesitant fuzzy sets are necessary, and are obtained adapting the ones given for finitely generated sets in Definition 2.5.

Definition 2.8 Let $X$ be a non-empty set, and $A, B \in I V H F S(X)$. Then:

- $A \vee B$ is given by the membership function $\mu_{A \vee B}$, such that for each $x \in X, \mu_{A \vee B}(x)=$ $\mu_{A}(x) \vee \mu_{B}(x)$, 
- $A \wedge B$ is given by the membership function $\mu_{A \wedge B}$, such that for each $x \in X, \mu_{A \wedge B}(x)=$ $\mu_{A}(x) \wedge \mu_{B}(x)$.

The next finite interval-valued hesitant fuzzy set will be necessary in the axiomatic definitions of cardinality.

Definition 2.9 Given $X$ a non-empty set, $a \in$ $F G([0,1])$ and $x \in X$, the set $a / x \in \operatorname{IVHFS}(X)$ is given by the membership function $\mu_{a / x}$ where $\mu_{a / x}(x)=a$ and $\mu_{a / x}(y)=0, \forall y \neq x$.

In the following remark, particular cases for fuzzy sets and interval-valued fuzzy sets are given.

Remark 2.10 Given $a / x \in I V H F S(X)$ as in the previous definition,

- if $a \subset[0,1]$, then $a / x \in F S(X)[17]$,

- if $a \subset L([0,1])$, then $a / x \in I V F S(X)[5]$.

An ordering relation for finite interval-valued hesitant fuzzy sets is also necessary in the following section for the development of cardinality of this type of sets. Several orders have been defined by Pérez et al. in [9]. In our work, the chosen ordering relation is related to $\mathrm{Xu}$ and Yager (see [18]) total ordering relation for intervals, which is given next, before than the developed ordering relation for finite interval-valued hesitant fuzzy sets is presented.

Definition 2.11 Given $\mathbf{x}=\left[x^{L}, x^{U}\right], \quad \mathbf{y}=$ $\left[y^{L}, y^{U}\right] \in L([0,1])$, and the score and accuracy functions $S(\mathbf{x})=x^{U}-x^{L}$ and $H(\mathbf{x})=x^{L}+x^{U}$ respectively. Then, the total ordering relation $\leq_{X Y}$ is given as follows:

$\mathbf{x} \leq_{X Y} \mathbf{y} \Longleftrightarrow\left\{\begin{array}{l}H(\mathbf{x})<H(\mathbf{y}), \\ o r \\ H(\mathbf{x})=H(\mathbf{y}) \text { and } S(\mathbf{x})<S(\mathbf{y}) .\end{array}\right.$

In the following definition, the used ordering relation for finite interval-valued hesitant fuzzy sets is given, which has been proven to be an ordering relation in our previous paper (see [10]).

Definition 2.12 Let $X$ be a non-empty set with cardinality $N, A, B \in I V H F S(X)$ such that $\forall x \in$ $X$ :

$$
\begin{aligned}
& \mu_{A}(x)=\bigcup_{i=1}^{n_{x}^{A}} A_{i}^{x}=\bigcup_{i=1}^{n_{x}^{A}}\left[A_{i}^{x^{L}}, A_{i}^{x^{U}}\right], \\
& \mu_{B}(x)=\bigcup_{i=1}^{n_{x}^{B}} B_{i}^{x}=\bigcup_{i=1}^{n_{x}^{B}}\left[B_{i}^{x^{L}}, B_{i}^{x^{U}}\right],
\end{aligned}
$$

where for simplicity and without loss of generality it is supposed that the sets are ordered increasingly, i.e., $A_{i}^{x} \leq_{X Y} A_{i+1}^{x}, B_{i}^{x} \leq_{X Y} B_{i+1}^{x}$, and $A_{i}^{x} \cap A_{j}^{x}=\emptyset$ and $B_{i}^{x} \cap B_{j}^{x}=\emptyset$.
Given $I \in F G([0,1])$, and $S$ and $H$ the following functions (score and accuracy, respectively):

$$
S(I)=\sum_{i=1}^{n_{I}}\left(I_{i}^{U}-I_{i}^{L}\right), H(I)=\frac{1}{n_{I}} \sum_{i=1}^{n_{I}}\left(\frac{I_{i}^{L}+I_{i}^{U}}{2}\right),
$$

then, $A \leq B$ if only if

(a) $H\left(\mu_{A}(x)\right) \leq H\left(\mu_{B}(x)\right) \forall x \in X$ and $\exists x^{\prime}$ s. t. $H\left(\mu_{A}\left(x^{\prime}\right)\right)<H\left(\mu_{B}\left(x^{\prime}\right)\right)$ or

(b) $H\left(\mu_{A}(x)\right)=H\left(\mu_{B}(x)\right) \forall x \in X$ and

(b1) $S\left(\mu_{A}(x)\right) \leq S\left(\mu_{B}(x)\right) \forall x \in X$ and $\exists x^{\prime}$ s. t. $S\left(\mu_{A}\left(x^{\prime}\right)\right)<S\left(\mu_{B}\left(x^{\prime}\right)\right)$ or

(b2) $S\left(\mu_{A}(x)\right)=S\left(\mu_{B}(x)\right) \forall x \in X$ and

$$
\begin{aligned}
\text { (b2.1) } & n_{x}^{A} \leq n_{x}^{B} \forall x \in X \text { and } \\
& \exists x^{\prime} \text { s. } t . n_{x^{\prime}}^{A}<n_{x^{\prime}}^{B} \text { or } \\
\text { (b2.2) } & n_{x}^{A}=n_{x}^{B}, A_{i}^{x^{U}} \leq B_{i}^{x^{U}} \text { and } \\
& A_{i}^{x^{L}} \leq B_{i}^{x^{L}}, \forall x \in X, \forall i=1, \ldots, n_{x}^{A} .
\end{aligned}
$$

One of the main interests of the finite intervalvalued hesitant fuzzy sets lies on the fact that they generalize fuzzy sets and interval-valued fuzzy sets.

The last part of this section is related to the cardinality of fuzzy sets. The capability to count elements in a set is straightforward when working with crisp sets. However, this task is harder to carry out when we are dealing with another types of sets, such as fuzzy sets or interval-valued fuzzy sets.

For this reason, several authors have developed different ways to measure the cardinality of these types of sets. Some proposals for fuzzy sets have been tried, such as $\sigma$-count, given by De Luca and Termini (see [6]).

Definition 2.13 Let $X=\left\{x_{1}, \ldots, x_{N}\right\}$ and $A \in$ $F S(X)$ be two sets, the $\sigma$-count cardinality of $A$ is given by:

$$
|A|_{\sigma}=\sum_{i=1}^{N} \mu_{A}\left(x_{i}\right) .
$$

However, this cardinality is hard to understand, as most of the time the obtained value will be a non integer number.

One of the most important attempts to achieve the cardinality of fuzzy sets has been given by Ralescu (see [12]), where he shows a non fuzzy cardinality for this type of sets. In the next definition, the original proposal is given as in [12].

Definition 2.14 Let $X=\left\{x_{1}, \ldots, x_{N}\right\}$ be a set and $A$ a $F S(X)$ where $\mu_{A}$ is its membership function, the values $\mu_{A}\left(x_{1}\right), \ldots, \mu_{A}\left(x_{N}\right)$ are ordered decreasingly, where $\mu_{(i)}$ denotes the $i$-th largest value, such that:

$$
1=\mu_{(0)} \geq \mu_{(1)} \geq \cdots \geq \mu_{(N)} \geq \mu_{(N+1)}=0 .
$$

Then, the non-fuzzy cardinality is given by:

$$
|A|_{R}= \begin{cases}0, & \text { if } A=\emptyset, \\ j, & \text { if } A \neq \emptyset \text { and } \mu_{(j)} \geq 0.5, \\ j-1, & \text { if } A \neq \emptyset \text { and } \mu_{(j)}<0.5 .\end{cases}
$$


where $j=\max \left\{1 \leq t \leq N \mid \mu_{(t-1)}+\mu_{(t)}>1\right\}$.

In the same work, Ralescu proves the next result that will be useful in our paper.

Proposition 2.15 Let $X=\left\{x_{1}, \ldots, x_{N}\right\}$ be a set and let $A, B$ two $F S(X)$, then:

$$
|A \cup B|_{R}=|A|_{R}+|B|_{R}-|A \cap B|_{R},
$$

where $|\cdot|_{R}$ represents the non-fuzzy cardinality given in Definition 2.14.

This cardinality has been applied in different works, such as in [11], where an approach to protect the privacy of microdata using fuzzy partitions is developed.

Another remarkable definition of cardinality for fuzzy sets has been given by Wygralak (see [17]), where instead of giving a fixed function as Ralescu did, he proposed an axiomatic definition of cardinality.

Definition 2.16 Let $X=\left\{x_{1}, \ldots, x_{N}\right\}$ be a set, the mapping $|\cdot|: F S(X) \rightarrow[0, \infty)$ is a scalar cardinality measure for fuzzy sets if it satisfies the following properties, given $A, B \in F S(X), x, y \in X$ and $a, b \in[0,1]$ :

$$
\begin{aligned}
& \text { 1. }|1 / x|=1 \text {, (coincidence) } \\
& \text { 2. } a \leq b \Rightarrow|a / x| \leq|b / y| \text {, (monotonicity) } \\
& \text { 3. }|A \cup B|=|A|+|B| \text { if } A \cap B=\emptyset \text {. (additivity) }
\end{aligned}
$$

It is easy to prove that Ralescu non-fuzzy cardinality and De Luca and Termini $\sigma$-count are Wygralak scalar cardinalities.

There are other definitions of cardinality for fuzzy sets (such as the one provided by [19]). However, in this subsection we only described the ones needed for a better understanding of the work.

In the following section, a new proposal is given to measure the cardinality of finite interval-valued hesitant fuzzy sets in a similar way as the scalar cardinality given by Wygralak. Furthermore, results that allow us to relate them with the most usual cardinalities for fuzzy sets when restricted to them are also given.

\section{Cardinality for finite interval-valued hesitant fuzzy sets}

One of the topics related to finite interval-valued hesitant fuzzy sets that, to the best of our knowledge, has not been dealt with yet is the cardinality. Obviously, due to the definition of the membership functions, this is a more difficult approach than with crisp or fuzzy sets.

Our aim in this section is to tackle this problem, with an axiomatic definition of cardinality for finite interval-valued hesitant fuzzy sets. Particular cases will be shown too, and proven that they are closely related to $\sigma$-count and the non-fuzzy cardinality for fuzzy sets.

In the following definition, the axiomatic definition of cardinality for finite interval-valued hesitant fuzzy sets is given, with a similar structure to the one given by Wygralak in Definition 2.16.

Definition 3.1 Let $X=\left\{x_{1}, \ldots, x_{N}\right\}$ be a set, the mapping $|\cdot|: \operatorname{IVHFS}(X) \rightarrow[0, \infty)$ is a scalar cardinality measure for finite interval-valued hesitant fuzzy sets if it satisfies the following properties, given $A, B \in \operatorname{IVHFS}(X), x, y \in X$ and $a, b \in F G([0,1]):$

1. $|1 / x|=1$, (coincidence)

2. $a \leq b \Rightarrow|a / x| \leq|b / y|$, (monotonicity)

3. $|A \vee B|=|A|+|B|$ if $A \wedge B=\emptyset$, (additivity)

where $\vee$ and $\wedge$ are given by Definition 2.8.

Remark 3.2 It must be noted that the ordering relation used for finitely generated sets in the second axiom is the one given in Definition 2.12 when $X$ is a singleton.

Some important properties that scalar cardinalities for finite interval-valued hesitant fuzzy sets satisfy are shown in the next result. Note that if $A, B$ are $I V H F S$, then their union (intersection) is an IVHFS set, which values at $\mathrm{x}$ are the unions (intersections) derived from Definition 2.5.

Proposition 3.3 Let $X=\left\{x_{1}, \ldots, x_{N}\right\}$ be a set and let $|\cdot|: \operatorname{IVHFS}(X) \rightarrow[0, \infty)$ be a scalar cardinality measure for finite interval-valued hesitant fuzzy sets. Then, it satisfies the following properties:

(i) Given $A_{1}, \ldots, A_{n} \in \operatorname{IVHFS}(X)$ such that $A_{i} \wedge A_{j}=\emptyset, \forall i \neq j$. Then,

$$
\left|\bigvee_{i=1}^{n} A_{i}\right|=\sum_{i=1}^{n}\left|A_{i}\right| .
$$

(ii) Given $A, B \in I V H F S(X)$ such that $A \leq B$, then $|A| \leq|B|$.

(iii) If $A$ is a crisp set, then $|A| \in \mathbb{N}$.

(iv) Given $x, y \in X$ and $a \in F G([0,1])$, then $|a / x|=|a / y|$.

(v) Given $A, B \in I V H F S(X)$ and a bijection $\sigma$ : $\{1, \ldots, N\} \rightarrow\{1, \ldots, N\}$ such that $\mu_{A}\left(x_{i}\right)=$ $\mu_{B}\left(x_{\sigma(i)}\right), \forall i \in\{1, \ldots, N\}$, then $|A|=|B|$.

(vi) $|\emptyset|=0$ and $|X|=N$.

(vii) $0 \leq|A| \leq N$

In the next theorem, a simplification is proposed in order to obtain scalar cardinalities in an easier way than the original definition. 
Theorem 3.4 Let $X=\left\{x_{1}, \ldots, x_{N}\right\}$ be a set, the mapping $|\cdot|: \operatorname{IVHFS}(X) \rightarrow[0, \infty)$ is a scalar cardinality for finite interval-valued hesitant fuzzy sets if and only if it exists a mapping $f: F G([0,1]) \rightarrow$ $[0,1]$ that satisfies the following properties:

1. $f(\{0\})=0$ and $f(\{1\})=1$,

2. given $a, b \in F G([0,1])$ such that $a \leq b$, then $f(a) \leq f(b)$,

such that for every $A \in I V H F S(X)$ :

$$
|A|=\sum_{i=1}^{N} f\left(\mu_{A}\left(x_{i}\right)\right)
$$

Proof Firstly, let us suppose that $|\cdot|$ is a scalar cardinality. Let us define the mapping $f: F G([0,1]) \rightarrow$ $[0,1]$, where $f(a)=|a / x|$, with $a \in F G([0,1])$ and whichever $x \in X$ (as it does not matter this choice by property (iv) in Proposition 3.3).

As any set $A \in I V H F S(X)$ can be decomposed as $A=\bigvee_{i=1}^{N} \mu_{A}\left(x_{i}\right) / x_{i}$, by property $(i)$ of Proposition 3.3

$$
\begin{aligned}
|A| & =\left|\bigvee_{i=1}^{N} \mu_{A}\left(x_{i}\right) / x_{i}\right|=\sum_{i=1}^{N}\left|\mu_{A}\left(x_{i}\right) / x_{i}\right|= \\
& =\sum_{i=1}^{N}\left|\mu_{A}\left(x_{i}\right) / x\right|=\sum_{i=1}^{N} f\left(\mu_{A}\left(x_{i}\right)\right) .
\end{aligned}
$$

Therefore, it is enough to see that $f$ satisfies the two properties of the theorem:

1. Applying property (vi) of Proposition 3.3 and the first axiom of scalar cardinality respectively:

$$
\begin{aligned}
& f(\{0\})=|0 / x|=|\emptyset|=0, \\
& f(\{1\})=|1 / x|=1 .
\end{aligned}
$$

2. Given $a, b \in F G([0,1])$ such that $a \leq b$, then by the second axiom of scalar cardinality, $|a / x| \leq$ $|b / x|$, and by definition of $f, f(a) \leq f(b)$.

In the second part of the proof it is supposed that it exists a mapping $f$ as defined in the theorem. To see that $|\cdot|$ is a scalar cardinality, the three axioms of Definition 3.1 must be proven.

1. Given $x \in X$ :

$$
\begin{aligned}
|1 / x| & =\sum_{i=1}^{N} f\left(\mu_{1 / x}\left(x_{i}\right)\right)= \\
& =(N-1) f(\{0\})+f(\{1\})=1,
\end{aligned}
$$

2. Given $x, y \in X$, and $a, b \in F G([0,1])$ such that $a \leq b$, by hypothesis, $f(a) \leq f(b)$. By defini- tion of the sets $|a / x|$ and $|b / y|$ :

$$
\begin{aligned}
& |a / x|=\sum_{i=1}^{N} f\left(\mu_{a / x}\left(x_{i}\right)\right)=f(a) \leq \\
& \leq f(b)=\sum_{i=1}^{N} f\left(\mu_{b / y}\left(x_{i}\right)\right)=|b / y| .
\end{aligned}
$$

3. Let $A, B \in I V H F S(X)$ such that $A \wedge B=\emptyset$. By Proposition 2.6, it is known that $\mu_{A}(x)=\emptyset$ or $\mu_{B}(x)=\emptyset$ for every $x \in X$, which means that the intersection of the support is null, where the support is denoted and defined as $\operatorname{Supp}(A)=\left\{x \in X \mid \mu_{A}(x) \neq\{0\}\right\}$. In our case, $\operatorname{Supp}(A) \cap \operatorname{Supp}(B)=\emptyset$ and $\operatorname{Supp}(A \vee B)=$ $\operatorname{Supp}(A) \cup \operatorname{Supp}(B)$. Thus:

$$
\begin{aligned}
|A \vee B|= & \sum_{x \in \operatorname{Supp}(A \vee B)} f\left(\mu_{A \cup B}(x)\right)= \\
= & \sum_{x \in \operatorname{Supp}(A)} f\left(\mu_{A}(x)\right)+ \\
& +\sum_{x \in \operatorname{Supp}(B)} f\left(\mu_{B}(x)\right)= \\
= & |A|+|B|,
\end{aligned}
$$

as for every $x \notin \operatorname{Supp}(A), \mu_{A}(x)=\{0\}$ and as a result $f\left(\mu_{A}(x)\right)=0$. Respectively for $B$ and $A \vee B$.

As it has been previously said, this result provides an alternative way to obtain a scalar cardinality, just with a mapping satisfying the two axioms. An example obtained from this last theorem is given next.

Example 3.5 Given the set $X=\left\{x_{1}, \ldots, x_{N}\right\}$, the mapping $|\cdot|: \operatorname{IVHFS}(X) \rightarrow[0, \infty)$ given such that for every $A \in I V H F S(X)$ :

$$
|A|=\sum_{i=1}^{N} H\left(\mu_{A}\left(x_{i}\right)\right),
$$

where $H$ is the accuracy function given in Definition 2.12, is a scalar cardinality for finite interval-valued hesitant fuzzy sets.

Remark 3.6 It must be noted that the previous example, when it is restricted to fuzzy sets, matches the $\sigma$-count cardinality.

This axiomatic definition allows to classify a wide range of functions as cardinalities for finite intervalvalued hesitant fuzzy sets, avoiding the restriction to a fixed one. However, a particular case will be highlighted in this section due to the special properties that it presents when it is restricted to fuzzy sets.

Firstly, a way to obtain a fuzzy set from a finite interval-valued hesitant fuzzy set using the accuracy function given in Definition 2.12 is presented next. Furthermore, a result related to this definition is given right after. 
Definition 3.7 Let $A$ be a $\operatorname{IVHFS}(X)$, the fuzzy set $A^{\prime}=\left\{\left(x, \mu_{A^{\prime}}(x)\right) \mid x \in X\right\}$ obtained from $A$ is defined as:

$$
\mu_{A^{\prime}}(x)=H\left(\mu_{A}(x)\right), \forall x \in X=\left\{x_{1}, \ldots, x_{N}\right\},
$$

where $H$ represents the accuracy function given in Definition 2.12.

Proposition 3.8 Given $A, B \in I V H F S(X)$ such that $A \wedge B=\emptyset$, then:

$$
(A \vee B)^{\prime}=A^{\prime} \cup B^{\prime}
$$

In the following example, the aforementioned particular case is shown.

Example 3.9 Given the sets $X=\left\{x_{1}, \ldots, x_{N}\right\}$, $A \in I V H F S(X)$ where

$$
\mu_{A}\left(x_{i}\right)=\bigvee_{j=1}^{n_{x_{i}}} A_{j}^{x_{i}}=\bigvee_{j=1}^{n_{x_{i}}}\left[A_{j}^{x_{i}^{L}}, A_{j}^{x_{i}^{U}}\right], \forall i=1, \ldots, N
$$

and $A^{\prime} \in F S(X)$ obtained as in Definition 3.7, the finitely generated sets $\mu_{A^{\prime}}\left(x_{i}\right) \forall i=1, \ldots, N$, are ordered decreasingly, where $\mu_{(i)}$ denotes the $i$-th largest value such that:

$$
1=\mu_{(0)} \geq \mu_{(1)} \geq \cdots \geq \mu_{(N)} \geq \mu_{(N+1)}=0 .
$$

Then, the function $|\cdot|: \operatorname{IVHFS}(X) \rightarrow[0, \infty)$ given by:

$$
|A|= \begin{cases}0, & \text { if } A=\emptyset \\ j, & \text { if } A \neq \emptyset \text { and } \mu_{(j)} \geq 0.5 \\ j-1, & \text { if } A \neq \emptyset \text { and } \mu_{(j)}<0.5\end{cases}
$$

where

$$
j=\max \left\{1 \leq t \leq N \mid \mu_{(t-1)}+\mu_{(t)}>1\right\},
$$

is a scalar cardinality for finite interval-valued hesitant fuzzy sets.

Remark 3.10 It is immediate to see that the scalar cardinality $|\cdot|$ given in Example 3.9 restricted to fuzzy sets matches Ralescu's cardinality for fuzzy sets (Definition 2.14), as given $A \in F S(X)$, $H\left(\mu_{A}\left(x_{i}\right)\right)=\mu_{A}(x) \forall x \in X$, so $A^{\prime}=A$, and the remainder of the process is the same. It also must be noted that $|A|=\left|A^{\prime}\right|_{R}$.

\section{Conclusions}

Along this work, the definition of cardinality for finite interval-valued hesitant fuzzy sets has been tackled from an axiomatic point of view. Furthermore, different results have been developed around this definition. From this perspective, cardinality is not fixed to a single function, but a wide range of mappings are considered as cardinalities.

It has also been shown the close relation of this definition with more usual ones in the fuzzy logic.
The own axiomatic definition is the one given by Wygralak for fuzzy sets. On the other hand, and supported by all the results given, two examples has been given. These examples, when restricted to fuzzy sets, are the well known fuzzy cardinalities $\sigma$-count and non-fuzzy cardinality.

This axiomatic definition allows to measure the cardinality of a finite interval-valued hesitant fuzzy set, remaining close to the most well known cardinalities in other type of sets, thanks to the generalization that finite interval-valued hesitant fuzzy sets provide.

\section{References}

[1] B. Bedregal, R. Santiago, H. Bustince, D. Paternain, R. Reiser, Typical Hesitant Fuzzy Negations, International Journal of Intelligent Systems 26 (6) (2014), 525-543.

[2] B. Bedregal, R. Reiser, H. Bustince, C. LopezMolina, V. Torra, Aggregation functions for typical hesitant fuzzy elements and the action of automorphisms, Information Sciences 255 (10) (2014), 82-99.

[3] S. Chen, J. Hong, Multicriteria linguistic decision making based on hesitant fuzzy linguistic term sets and the aggregation of fuzzy sets, Information Sciences 286 (2014), 63-74.

[4] N. Chen, Z.S. Xu, M.M. Xia, Interval-valued hesitant preference relations and their applications to group decision making, KnowledgeBased Systems 37 (1) (2013), 528-540.

[5] G. Deschrijver, P. Král On the cardinalities of interval-valued fuzzy sets, Fuzzy Sets and Systems 158(15) (2007), 1728-1750.

[6] A. De Luca, S. Termini, A definition of nonprobabilistic entropy in the setting of fuzzy set theory, Information and Control 20 (1972), 301312.

[7] I. Grattan-Guinness, Fuzzy membership mapped onto interval and many-valued quantities, Zeitschrift für mathematische Logik und Grundladen der Mathematik 22 (1976), 149-160.

[8] N.R. Pal, H. Bustince, M. Pagola, U.K. Mukherjee, D.P. Goswami, G. Beliakov, Uncertainties with Atanassov's intuitionistic fuzzy sets: Fuzziness and lack of knowledge, Information Sciences 97 (2013), 61-74.

[9] R. Pérez, P. Alonso, H. Bustince, I. Díaz, A. Jurio, S. Montes, Ordering finitely generated sets and finite interval-valued hesitant fuzzy sets, submitted.

[10] P. Quirós, P. Alonso, H. Bustince, I. Díaz, S. Montes, An entropy measure definition for interval-valued hesitant fuzzy sets, submitted.

[11] P. Quirós, P. Alonso, I. Díaz, S. Montes, Protecting data: a fuzzy approach, International Journal of Computer Mathematics, in press. DOI:10.1080/00207160.2014.928700. 
[12] D. Ralescu, Cardinality, quantifiers, and the aggregation of fuzzy criteria, Fuzzy Sets and Systems 69 (1995), 355-365.

[13] R.M. Rodríguez, L. Martínez, V. Torra, Z.S. $\mathrm{Xu}, \mathrm{F}$. Herrera, Hesitant Fuzzy Sets: State of the Art and Future Directions, International Journal of Intelligent Systems 29 (6) (2014), 495-524.

[14] R. Sambuc, Fonctions $\Phi$-floues. Application l'Aide au Diagnostic en Patholologie Thyroidienne, Ph. D. Thesis, Univ. Marseille (1975).

[15] V. Torra, Y. Narukawa, On hesitant fuzzy sets and decision, Proceedings of FUZZ-IEEE (2009), 1378-1382.

[16] V. Torra, Hesitant fuzzy sets, International Journal of Intelligent Systems 25 (2010), 529539.

[17] M. Wygralak, Cardinalities of Fuzzy Sets, Springer-Verlag Berlin Heidelberg (2003), DOI 10.1007/978-3-540-36382-8.

[18] Z. Xu, R.R. Yager, Some geometric aggregation operators based on intuitionistic fuzzy sets, International Journal of General Systems 35 (2006), 417-433.

[19] R.R. Yager, Cardinality of fuzzy sets via bags, Mathematical Modelling 9 (6) (1987), 441-446.

[20] L. Zadeh, Similarity relations and fuzzy orderings, Information Sciences 3 (1971), 177-200.

[21] L. Zadeh, The Concept of a Linguistic Variable and Its Application to Approximate Reasoning, Information Sciences 8 (1975), 199-249. 